\title{
A Gammaherpesvirus Establishes Persistent Infection in Neuroblastoma Cells
}

\author{
Hye-Jeong Cho, and Moon Jung Song*
}

\begin{abstract}
Gammaherpesvirus $(\gamma \mathrm{HV})$ infection of the central nervous system (CNS) has been implicated in diverse neurological diseases, and murine $\gamma \mathrm{HV}-68$ (MHV-68) is known to persist in the brain after cerebral infection. The underlying molecular mechanisms of persistency of virus in the brain are poorly understood. Here, we characterized a unique pattern of MHV-68 persistent infection in neuroblastoma cells. On infection with MHV-68, both murine and human neuroblastoma cells expressed viral lytic proteins and produced virions. However, the infected cells survived productive infection and could be cultured for multiple passages without affecting their cellular growth. Latent infection as well as productive replication was established in these prolonged cultures, and lytic replication was further increased by treatment with lytic inducers. Our results provide a novel system to study persistent infection of $\gamma \mathrm{HVs}$ in vitro following de novo infection and suggest application of MHV-68 as a potential gene transfer vector to the brain.
\end{abstract}

\section{INTRODUCTION}

Epstein-Barr virus (EBV) is considered as an important human virus owing to its association with diverse malignancies and diseases. EBV infection has been linked to various neurological diseases such as aseptic meningitis, encephalitis, human immunodeficiency virus (HIV)-related primary central nervous system (CNS) lymphoma, and multiple sclerosis (MS) (Bossolasco et al., 2006; Said et al., 1997; Serafini et al., 2007). Consistent with the neurological pathogenesis of human gammaherpesvirus $(\gamma \mathrm{HV})$, murine gammaherpesvirus-68 (MHV-68 or also called $\gamma \mathrm{HV}-68$; a murine homolog of EBV) has also been reported to infect the brain and replicate in it as well as in various cell types derived from the CNS (Cho et al., 2009; Terry et al., 2000). Furthermore, our recent studies by noninvasive bioluminescence imaging of a

Virus-Host Interactions Laboratory, Division of Biotechnology, Department of Biosystems and Biotechnology, College of Life Sciences and Biotechnology, Korea University, Seoul 136-713, Korea

*Correspondence: moonsong@ korea.ac.kr

Received 5 February, 2014; revised 25 June, 2014; accepted 25 June, 2014; published online 30 June, 2014

Keywords: CNS, gammaherpesvirus, gene delivery, neuroblastoma, persistent infection recombinant MHV-68 expressing the firefly luciferase (M3FL) suggested that MHV-68 may persist in the brain following cerebral infection (Kang et al., 2012). However, the underlying molecular mechanisms of viral persistency in the brain remain poorly understood.

Similar to infection by other herpesviruses, $\gamma \mathrm{HV}$ infection manifests in two distinct phases: productive (lytic) replication and nonproductive (latent) infection. During productive replication, viral genes are actively expressed in a highly ordered manner and viral genome is amplified to generate progeny viruses, all of which are required for the spread of virus. Productive, lytic infection often accompanies severe cytopathic effects (CPEs), eventually leading to the death of the infected cells. During latency, $\gamma \mathrm{HVs}$ express few viral genes, maintain viral genomes as nuclear episomes, and utilize cellular DNA polymerase for genome amplification. During latency, no infectious virus particles are produced, but the infected cells continue to proliferate. Latent viruses can re-enter the productive lytic cycle when latently infected cells are treated with lytic inducers such as tetradecanoyl phorbol acetate (TPA) and sodium butyrate. Both latent and lytic infections of $\gamma \mathrm{HVs}$ are believed to be important for viral persistency, because latent infection facilitates virus survival from host immune surveillance and lytic replication aids in persistent infection by replenishing its latent reservoir in the host (Hwang et al., 2008)

While the $\gamma \mathrm{HVs}$ replicate and persist in lymphocytes, they can infect other cell types for viral persistency, such as macrophages, lung epithelial cells, and dendritic cells, which have been reported to serve as a persistent reservoir for MHV-68 by supporting lifelong latency (Flano et al., 2000; 2003; Stewart et al., 1998; Weck et al., 1999). In contrast, cultured endothelial cells have been reported to sustain persistent ongoing productive infection with the extended viability of the infected cells, but not viral latency (Suarez and van Dyk, 2008). The brain is a potential site of viral persistency in vivo (Kang et al., 2012); therefore, it is notable that MHV-68 infection of murine neuroblastoma cells resulted in productive replication of MHV-68, but did not manifest any apparent signs of cell deaths during the course of infection (Cho et al., 2009). Here, we further characterized a unique pattern of MHV68 infection in both murine and human neuroblastoma cells. Our results may provide a novel system to study persistent infection of $\gamma \mathrm{HVs}$ in vitro and suggest a potential usage of MHV-68 as a gene delivery vector to the brain. 
A

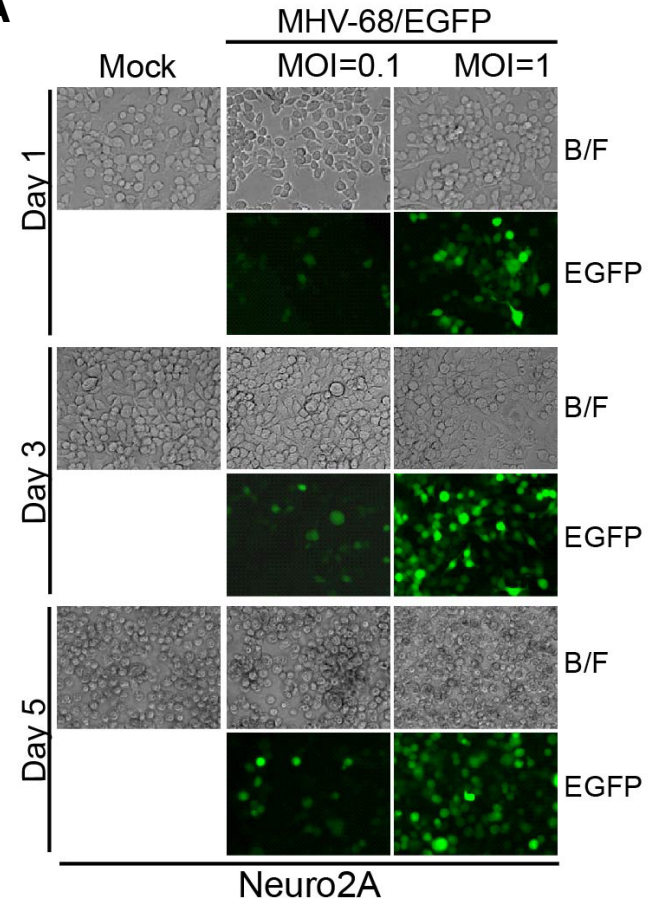

B
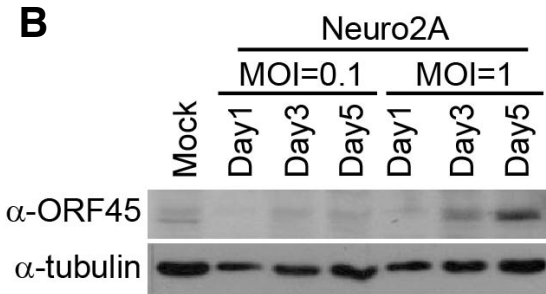

C
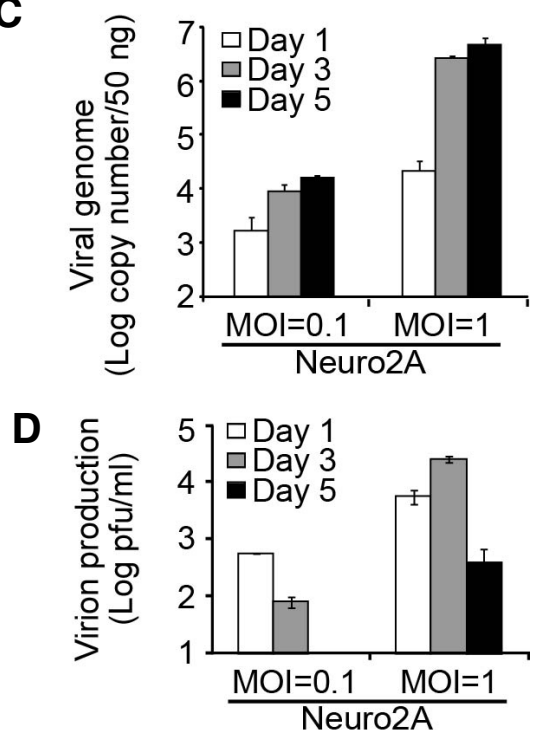

Fig. 1. Kinetic studies of MHV-68/EGFP infection in Neuro2A cells. Neuro2A, murine neuroblastoma cells, were infected with MHV-68/EGFP at an MOI of 0.1 or 1 and analyzed at 1,3 , and 5 days after infection for virus replication. (A) EGFP expression. The MHV-68/EGFP-infected and mock-infected Neuro2A cells were monitored for EGFP expression under a fluorescence microscope. The representative images are shown. B/F indicates the images from the bright field. (B) Viral lytic protein expression. The infected cell lysates were subjected to Western blotting with a polyclonal antibody against ORF45, a viral lytic protein. $\alpha$-Tubulin was used as the loading control. (C) Replication of viral genomes. The copy numbers of viral genomic DNAs extracted from infected cells were measured by real-time PCR. Three independent assays were performed in duplicate, and the means \pm standard deviation (SD) of the data are shown. (D) Production of infectious virions. The virus titers of the culture supernatants from the infected Neuro2A cells were measured by plaque assays. Three independent assays were performed, and the means $\pm S D$ of the data are shown. Mock-infected Neuro2A cells served as the negative control.

\section{MATERIALS AND METHODS}

Cells, viruses, and plaque assays

Neuro2A (a murine neuroblastoma cell line), SH-SY5Y (a human neuroblastoma cell line), BHK21 (a baby hamster kidney fibroblast cell line), and Vero (a green monkey kidney epithelial cell line) cells were propagated in complete Dulbecco's modified Eagle's medium supplemented with $10 \%$ fetal bovine serum and penicillin and streptomycin (10 units/ml) (HyClone). MHV-68 virus was originally obtained from the American Type Culture Collection (VR1465) and amplified in BHK21 cells as previously described (Lee et al., 2007). The viral titer was determined by plaque assays by using Vero monolayers overlaid with medium containing $1 \%$ methylcellulose (Lee et al., 2007).

\section{Antibodies, western blotting, and immunofluorescence} analysis

Cell lysates were analyzed with the following primary antibodies: rabbit polyclonal antibody to ORF26 (1:500), ORF45 (1:1000), or M9 (1:1000) and mouse monoclonal antibody to $\alpha$-tubulin (1:1000; Sigma). Goat anti-rabbit or goat anti-mouse IgG conjugated with horseradish peroxide secondary antibody (Santa
Cruz Biotechnology) was detected by using the enhanced peroxidase detection (EPD) Western Blot Detection Kit (ELPIS, Korea), and the signals were analyzed by using a chemiluminescent image analyzer (LAS-4000; Fujifilm). For immunofluorescence assays, the cells $\left(5 \times 10^{5}\right)$ were washed with $1 \times$ phosphate-buffered saline (PBS) and fixed for $40 \mathrm{~min}$ with $0.15 \%$ paraformaldehyde (Sigma). Then, the cells were incubated for $1 \mathrm{~h}$ at $4^{\circ} \mathrm{C}$ with anti-ORF45 or anti-M9 antibody (1:100) in $0.15 \%$ saponin solution (Sigma) and for $40 \mathrm{~min}$ at $4^{\circ} \mathrm{C}$ with Cy3-conjugated anti-rabbit IgG (1:200; Jackson Immune Research). Finally, the cells were analyzed under a fluorescence microscope (Zeiss).

\section{DNA extraction and real-time polymerase chain reaction (PCR) analysis}

Genomic DNAs including viral DNAs were isolated from the harvested cells by a standard method of phenol-chloroform extraction and ethanol precipitation (Lee et al., 2007). Real-time PCR of the whole genomic DNA (50 ng) was performed in duplicate on the iCycler iQ Multicolor Real-time PCR Detection System (Bio-Rad) using a $20-\mu l$ reaction mixture with ORF 56specific primers and SYBR green (Song et al., 2005). The PCR 

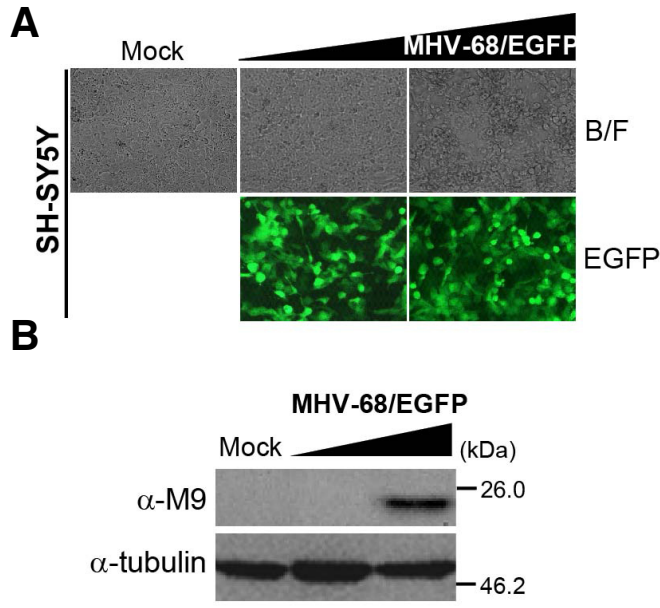

C

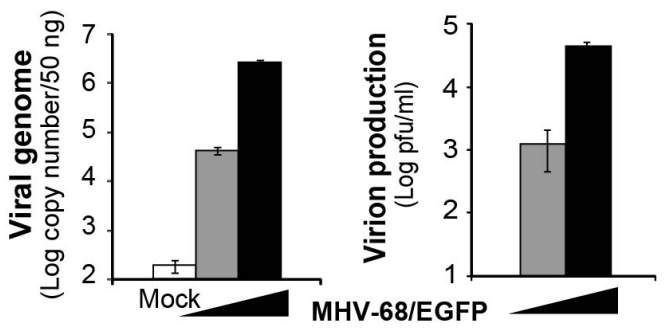

E

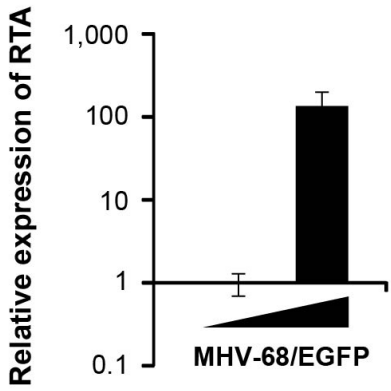

$\mathbf{F}$

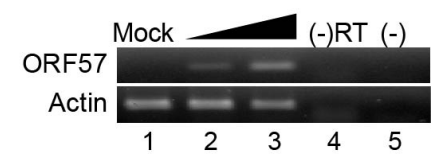

Fig. 2. Non-cytolytic, productive infection of MHV-68 in SH-SY5Y cells. SH-SY5Y, human neuroblastoma cells, were infected with MHV-68/EGFP at an MOI of 0.1 or 1 for 72 h. Mock-infected SH-SY5Y cells were used as the negative control. EGFP expression (A), M9 viral lytic protein expression (B), viral genome replication $(\mathrm{C})$, and virion production (D) from infected SH-SY5Y cells were analyzed as described in Fig. 1. Expression of an immediate early viral transcript, $R T A$, (E) and an early lytic transcript, ORF57, (F) were detected in SH-SY5Y cells by RT-qPCR and RT-PCR, respectively. Actin was used as an internal control for RTqPCR and RT-PCR.

was run at $50^{\circ} \mathrm{C}$ for $2 \mathrm{~min}$, followed by 45 cycles at $95^{\circ} \mathrm{C}$ for 10 $\mathrm{s}$, at $58^{\circ} \mathrm{C}$ for $15 \mathrm{~s}$, and $72^{\circ} \mathrm{C}$ for $20 \mathrm{~s}$, followed by the melting curve analysis.

\section{RNA extraction and reverse transcriptase (RT)-quantitative PCR (RT-qPCR)/RT-PCR analysis}

Total RNAs were extracted from cultured cells with TRI reagent (Molecular Research Center) according to the manufacturer's instructions. The cDNAs were synthesized by using the RevertAid First Strand cDNA Synthesis Kit (Fementas, Korea) with random hexamers. The synthesized $c D N A s$ were subjected to RT-qPCR or RT-PCR analysis with viral transcript-specific primers including RTA, ORF57, ORF29, and ORF73 or cellular $\beta$-actin-specific primers as described previously (Noh et al., 2012).

\section{Cell proliferation assays}

Cell viability was assayed via [3-(4,5-dimethylthiazol-2-yl) -2,5diphenyltetrazolium bromide] MTT assays as previously described (Cho et al., 2008). Briefly, $1 \times 10^{4}$ cells were seeded in $100 \mu \mathrm{l}$ of complete medium into each well of a 96 -well plate. At the indicated timepoints, $50 \mu \mathrm{l}$ of MTT solution $(2 \mathrm{mg} / \mathrm{ml})$ was added to each well, and the plate was incubated for an additional $4 \mathrm{~h}$ at $37^{\circ} \mathrm{C}$. The plates were then centrifuged, the supernatants were discarded, and $50 \mu \mathrm{l}$ of dimethyl sulfoxide was added to each well. Following dissolution of the crystals, the amount of reduced MTT was measured as absorbance at $570 \mathrm{~nm}$ using a $650-\mathrm{nm}$ reference.

\section{RESULTS AND DISCUSSION}

\section{Noncytolytic productive infection of MHV-68 in neuroblastoma cells}

In our previous study, we showed that MHV-68 productively replicated in Neuro2A cells without inducing severe CPEs (Cho et al., 2009). To validate this observation, we conducted a kinetic study of MHV-68 infection in Neuro2A cells for 5 days using a recombinant virus expressing the enhanced green fluorescence protein (MHV-68/EGFP) in this study (Fig. 1). The expression of EGFP and a viral lytic protein (ORF45, a tegument protein) in the infected Neuro2A cells increased in a timeand a dose-dependent manner (Figs. 1A and 1B). The copy numbers of the viral genome also increased in a similar manner (Fig. 1C). Furthermore, infectious virions were produced, albeit with different titers, depending on the time of harvesting the cells (Fig. 1E), indicating that Neuro2A cells genuinely supported productive infection of MHV-68 to completion. Although the infected cells tended to clump together more than the mock-infected cells, both mock- and virus-infected Neuro2A cells underwent morphological changes at day 5 post-infection (Fig. 1A). Despite the occurrence of productive virus replication, the levels of cell deaths in the infected Neuro2A cells were low and comparable to those in the mock-infected cells (data not shown). These results were consistent with our previous results in that the infected cells did not show apparent cell lysis and continued to express high level of EGFP.

To exclude the possibility of a cell line-specific phenomenon, we infected MHV-68/EGFP in SH-SY5Y, which is dopamine $\beta$ hydroxylase active, acetylcholinergic, glutamatergic, and adenosinergic (Bae et al., 2012; Kovalevich and Langford, 2013; Ross et al., 1983). Consistent with the previous results in 


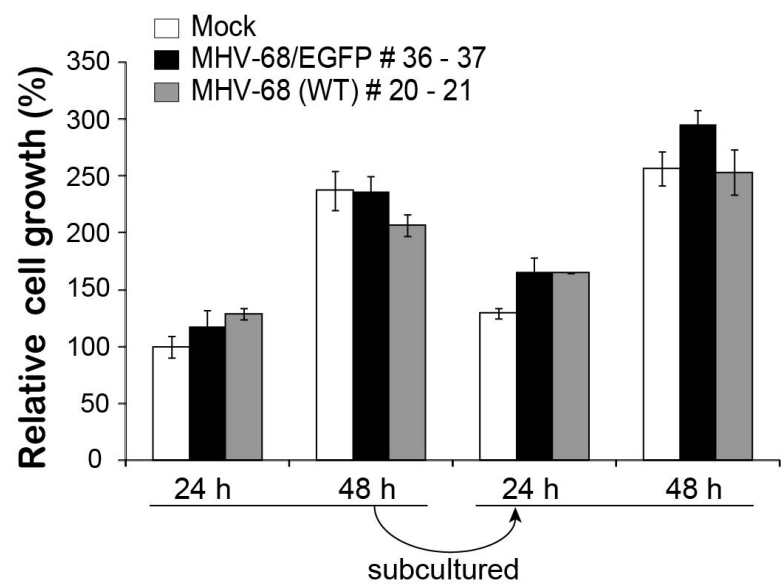

Fig. 3. Effect of MHV-68 infection on the relative growth of $\mathrm{SH}$ SY5Y cells. SH-SY5Y cells infected with MHV-68/EGFP or MHV-68 WT were subcultured for 36 passages or 20 passages, respectively. The infected cells were seeded in 96-well plates, cultured for $24 \mathrm{~h}$ and $48 \mathrm{~h}$, and analyzed for cell viability by MTT assays. The cells were subcultured at 1:10 ratio and the cell viability was monitored for another 24 and $48 \mathrm{~h}$. The relative cell growth was calculated based on the cell viability of mock-infected SH-SY5Y cells at $24 \mathrm{~h}$ after the first seeding. Three sets of samples were used in each experiment, and the experiments were independently performed twice. The means \pm SD of the data are shown.

A

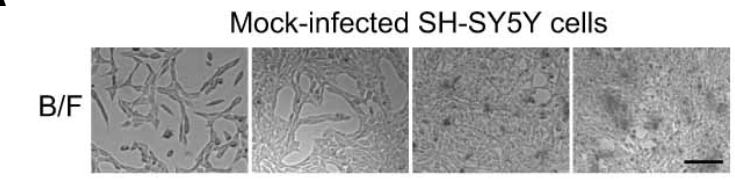

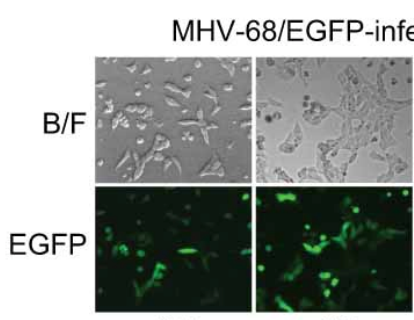

$24 \mathrm{~h}$

$48 \mathrm{~h}$
C

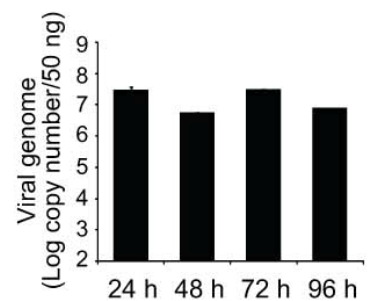

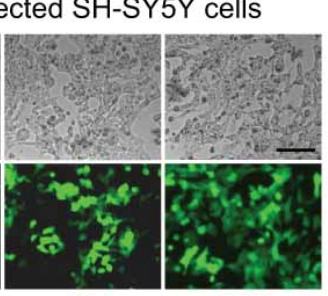

$72 \mathrm{~h}$

$96 \mathrm{~h}$
D

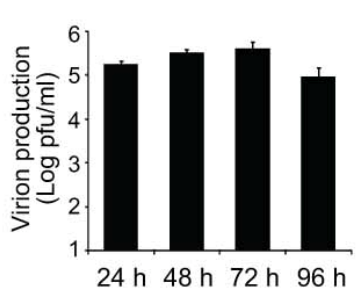

Neuro2A cells, SH-SY5Y cells successfully supported productive replication of MHV-68 without any apparent cell lysis (Fig. 2). The infected cells exhibited strong EGFP expression (Fig. $2 A)$ and expressed a viral lytic protein (M9, a small capsid protein) (Fig. 2B). Both viral genome replication and virion production increased in a dose-dependent manner (Figs. 2C and 2D). The viral transcripts such as RTA (an immediate early gene) and ORF57 (an early gene) were well-expressed in infected SH-SY5Y cells, as measured by RT-qPCR and RT-PCR, respectively (Figs. $2 \mathrm{E}$ and $2 \mathrm{~F}$ ). The infected cells continued to grow and did not show significant levels of cell deaths as compared with mock-infected cells (Fig. 2A). The same results were obtained with wild-type MHV-68 (WT) infection in both Neuro2A and SH-SY5Y cells (data not shown). These results suggest a unique pattern of infection in that MHV-68 productively replicates in both murine and human neuroblastoma cells without causing cell deaths. Since SH-SY5Y cells were easier to grow than Neuro2A cells even when uninfected, our subsequent studies focused on MHV-68 infection in SH-SY5Y cells.

\section{Persistent infection of MHV-68 in SH-SY5Y cells}

The WT- and MHV-68/EGFP-productive infections in both murine and human neuroblastoma cells resulted in minor morphological changes and little cell deaths at all multiplicity of infections (MOls) tested, and the cell continued to grow and become confluent; therefore, we split the infected cells at 1:10 ratio every alternate day for multiple passages. To examine the effect of MHV-68 infection on the growth of the infected cells, the
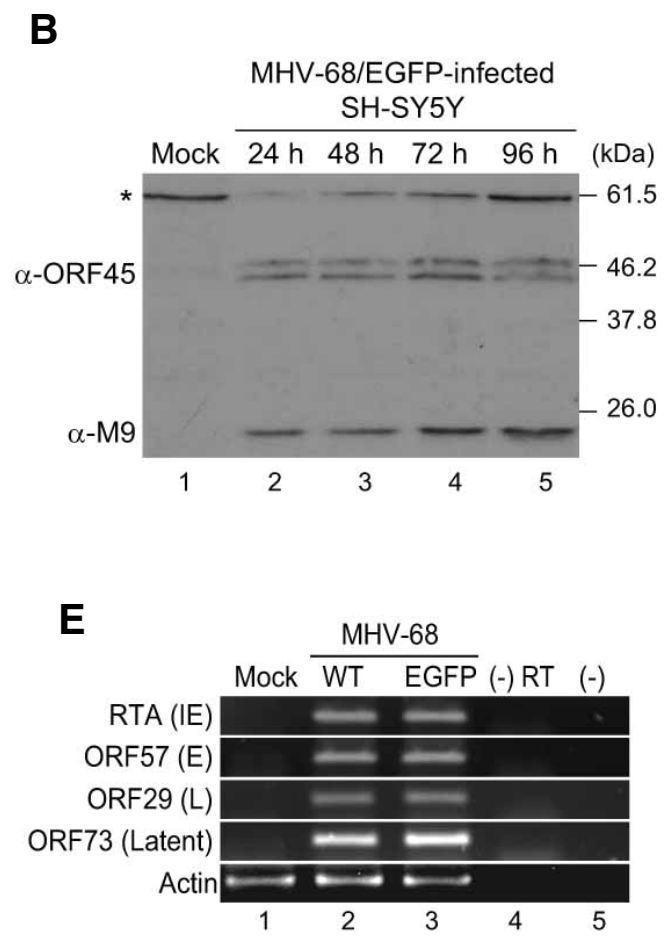

Fig. 4. Persistent infection of MHV-68/EGFP infection in SH-SY5Y cells cultured for multiple passages. (A-D) MHV-68/EGFP-infected SHSY5Y cells following 20 passages from the initial infection were monitored daily until $96 \mathrm{~h}$ after seeding. Expression of EGFP (A), viral lytic proteins, ORF45 and M9 (B), replication of viral genomic DNAs $(C)$, and virion production (D) from prolonged infection of SH-SY5Y cells were analyzed as described in Fig. 1. The asterisk symbol indicates a non-specific band (B). Expression of viral lytic and latent transcripts (E). SHSY5Y cells infected with MHV-68/EGFP or WT were cultured for 20 passages and harvested at $48 \mathrm{~h}$ post-seeding. Immediate-early (IE; RTA), early (E; ORF57), late (L; ORF29), and latent (ORF73) gene transcript were analyzed by RT-PCR. Actin was used as an internal control. Lanes 4 and 5 indicate negative controls without RT and template, respectively. 
A

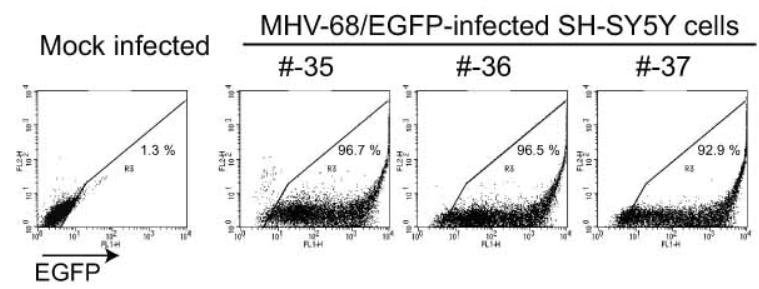

B
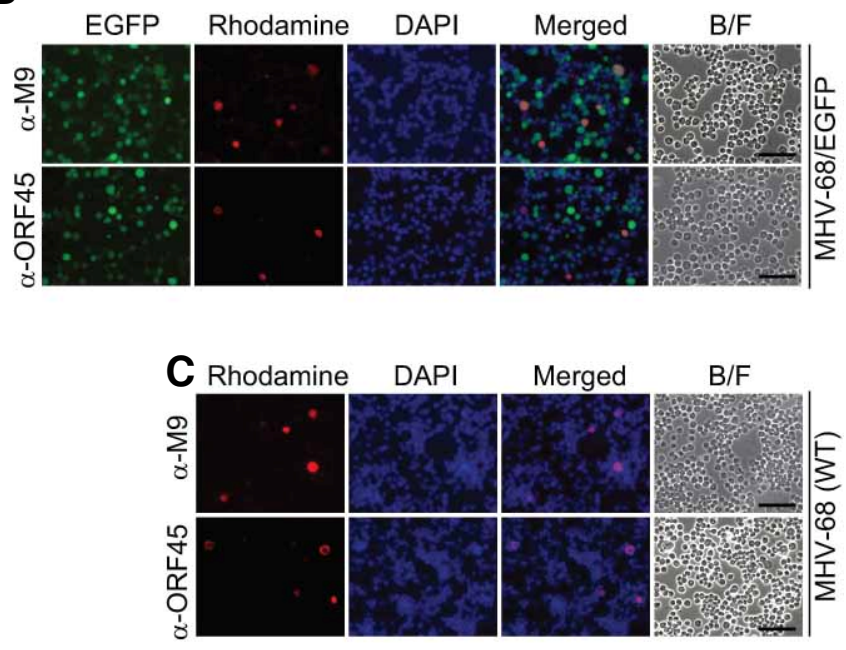

Fig. 5. Both productive and latent replications of MHV$68 /$ EGFP in prolonged culture of infected SH-SY5Y cells. (A) Persistent EGFP expression in the majority of MHV-68/ EGFP-infected SH-SY5Y cells. MHV-68/EGFP-infected SHSY5Y cells were grown for 35-37 passages from the initial infection and assayed by flow cytometry for EGFP expression. Mock-infected SH-SY5Y cells were used as the negative control. $(B, C)$ Sporadic expression of lytic viral proteins in the infected SH-SY5Y cells. MHV-68/EGFP- or WTinfected SH-SY5Y cells were grown for 34 or 20 passages from the initial infection, respectively. Immunofluorescence assays were perfor-med using antibodies against ORF45 and M9 viral lytic proteins in the infected cells under a fluorescence microscope. The representative images are shown. B/F indicates the images from the bright field. mock-, WT-, or MHV-68/EGFP-infected SH-SY5Y cells were seeded and subcultured at $48 \mathrm{~h}$ after the first seeding and grown for another $48 \mathrm{~h}$ (Fig. 3). The cells infected with either WT or MHV-68/EGFP grew at a similar rate to that with mock infection, suggesting that MHV-68 infection may not significantly alter the relative cellular growth of SH-SY5Y cells even with prolonged culturing.

Alternatively, MHV-68-infected SH-SY5Y cells may continue to grow with the gradual loss of viral genome after repetitive culturing of the infected cells. To test this possibility, the cells at the 20th passage were seeded and monitored daily for virus replication up to $96 \mathrm{~h}$ (Fig. 4). The subcultured SH-SY5Y cells sustained the ability to express EGFP and viral proteins and became similarly confluent as mock-infected cells (Figs. 4A and 4B). Viral genomic DNAs and infectious virions were consistently detected in the long-term culture of infected SH-SY5Y cells to the levels comparable to those of acutely infected SHSY5Y cells (Figs. 4C and 4D), indicating that the infected SHSY5Y cells supported persistent productive infection of MHV-68 rather than gradually losing the viral genome. On further analysis by RT-PCR of the viral gene expression in the SH-SY5Y cells persistently infected with MHV-68/EGFP or WT, all kinetic classes of lytic transcripts were detected, which confirmed ongoing lytic replication in the cells (Fig. 4E). Furthermore, the latent transcript of ORF73 was strongly expressed in the infected SH-SY5Y cells, suggesting that both lytic and latent infections may co-exist in these cultures.

Productive replication of MHV-68 maintained in a small population of the infected SH-SY5Y cells during persistent infection

MHV-68/EGFP-infected SH-SY5Y cells expressed relatively low levels of lytic transcripts, but strong latent transcripts (Fig. $4 \mathrm{E})$ probably due to inefficient productive infection in the most EGFP-positive cells or bona-fide productive replication only in a small subset of the population. To test this hypothesis, we examined EGFP and viral protein expressions at the single cell level (Fig. 5). More than $90 \%$ of the infected SH-SY5Y cells sustained EGFP expressions over the passages (passages 3537), as analyzed by flow cytometry analysis (Fig. 5A). Immunofluorescence assay results showed that approximately $5 \%$ of the cells expressed ORF45 and M9 among the EGFP-positive cells as well as WT-infected SH-SY5Y cells (Figs. 5B and 5C). Our results suggest that only a small population of the persistently infected neuroblastoma cells may support lytic infection of MHV-68, while the majority of the cells may harbor the MHV-68 genome with prolonged expression of the reporter gene. This observation suggests, at least in part, the characteristic feature of B cells latently infected with human $\gamma \mathrm{HVs}$, EBV, and Kaposi's sarcoma-associated herpesvirus (KSHV) that undergo low level of spontaneous lytic, productive replication. However, persistent replication of MHV-68 in the SH-SY5Y neuroblastoma cells significantly differs from that of EBV or KSHV in that the former yields infectious viral particles up to the titer of $10^{5} \mathrm{pfu} / \mathrm{ml}$, while human $\gamma \mathrm{HV}$ s produce few, if any, viral particles (Fig. 4D).

\section{Induction of MHV-68 productive replication in persistently} infected SH-SY5Y cells

To examine whether latent infection of MHV-68 in persistently infected SH-SY5Y cells can be further reactivated into productive lytic replication, the cells infected with MHV-68/EGFP or WT were treated with TPA and sodium butyrate $(\mathrm{NaB})$, which are well-known lytic inducers, in the presence and the absence of ganciclovir (GCV; a potent antiviral drug) for $36 \mathrm{~h}$. The RTA 

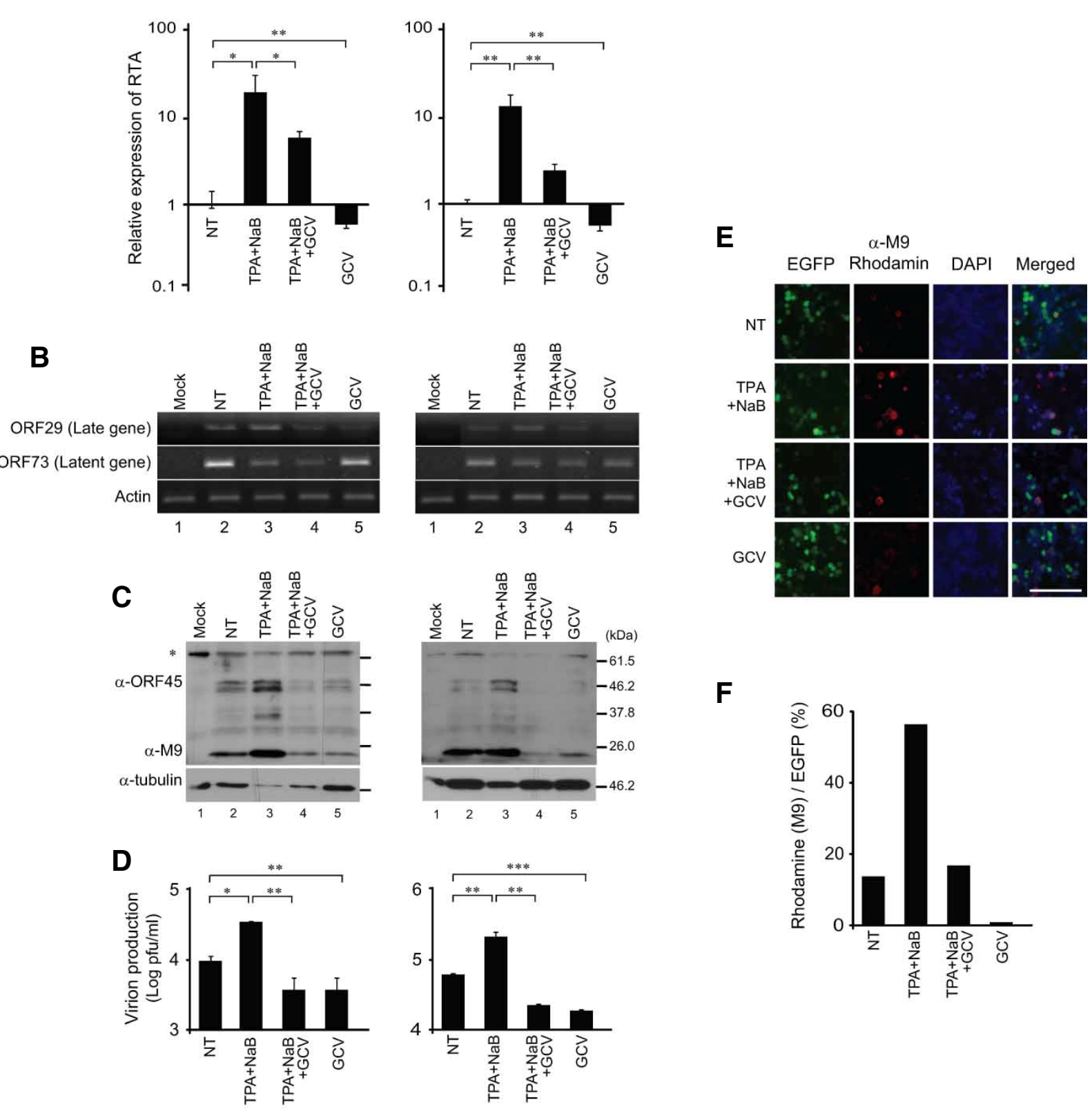

$$
{ }^{\star} p<0.05,{ }^{\star \star} p<0.01,{ }^{\star \star * \star} p<0.001
$$

Fig. 6. Induction of productive replication in prolonged culture of MHV-68/EGFP-infected SH-SY5Y cells. MHV-68/EGFP- and WT-infected SH-SY5Y cells were grown for 54 and 34 passages, respectively, and treated with TPA and NaB in the presence and absence of GCV for 36 h. (A) The relative expression of RTA transcript. The levels of RTA transcript measured by RT-qPCR. Actin was used as an internal control. The relative expression of the RTA transcript was calculated based on its level in the infected cells with no treatment (NT). (B) Expression of lytic and latent gene transcripts. The transcripts of a late gene (ORF29) and a latent gene (ORF73) were detected by RT-PCR. Actin was used as an internal control. (C) Expression of viral lytic proteins. The cell lysates were subjected to western blotting with antibodies against ORF45 and M9. Tubulin was used as the loading control; the asterisk symbol indicates the non-specific band. (D) Virion production. Virus titers were measured by plaque assays of the cultured supernatants harvested at $36 \mathrm{~h}$ after treatment. Statistical analysis was performed by Student's $t$ test (A, D). (E-F) Expression of a lytic protein determined by immunofluorescence assay. The MHV-68/EGFP-infected cells were subjected to immunofluorescence assays with the antibody against M9, a small capsid protein. The representative images are shown. A minimum of 100 EGFP-positive cells were counted for each sample, and the percentage of M9-positive cells among the EGFP-positive cells was calculated and shown in a graphical form $(F)$. 
transcript was induced by TPA and $\mathrm{NaB}$ treatments (Fig. 6A). TPA and $\mathrm{NaB}$ further increased the expression of other lytic transcripts and proteins, with concurrent increase in the production of infectious virions (Figs. 6B-6D). Immunofluorescence assay results indicated that higher number of cells was induced into productive replication rather than the same proportion of cells that express more lytic proteins (Figs. 6E and 6F). Meanwhile, the levels of the latent gene transcripts were inversely correlated with those of the lytic transcripts after treatment with TPA and NaB (Fig. 6B). The treatment with GCV inhibited lytic replication from both uninduced and induced $\mathrm{SH}-\mathrm{SY} 5 \mathrm{Y}$ cells, as evidenced by decreased viral lytic transcripts and proteins as well as reduced virion production (Fig. 6). Similar results were obtained from WT- and MHV-68/EGFP-infected SH-SY5Y cells. These results support the hypothesis that persistent infection of MHV-68 may be established in neuroblastoma cells in the form of both latent and productive replications.

An interesting feature of MHV-68 infection in neuroblastoma cells is that these cells support a prolonged expression of reporter genes. Gene transfer into the CNS is an emerging therapeutic strategy for various neurological diseases ranging from genetic disorders to brain cancer (Ralph et al., 2006). However, it is generally ineffective to deliver a gene through the bloodstream into the CNS because of the blood-brain barrier. Direct gene transfer into the brain has been attempted through intracranial injection, but it often leads to a limited transduction only in the region near the injected site, followed by subsiding expression of the delivered gene (Lentz et al., 2012). Several viral vectors derived from herpes simplex virus-1, adenovirus, adeno-associated virus, or retroviruses have been utilized to trans fer a gene of interest into the brain (Lentz et al., 2012). Although there have some cases of successful delivery, mostly, these viral vectors manifest the abovementioned limitations (Lentz et al., 2012; Ralph et al., 2006). Efficient gene transfer and prolonged gene expression are two important features required for effective gene delivery in the CNS. Our results suggest that MHV-68 may be an attractive candidate for the development of a gene transfer vector system. Considering that a functional genomic system as well as the bacterial artificial chromosome system is available in MHV-68 (Alder et al., 2001; Song et al., 2005; Wu et al., 2011), it is plausible to investigate the regions required to develop MHV-68 as a vector system. However, an efficient route of infection such as direct intracranial inoculation into the brain should be considered, because MHV-68 does not generally reach the brain by systemic infection. Nonetheless, to our knowledge, this is the first report suggesting MHV-68 as a potential gene-delivery vector in the brain, although further studies are warranted to ensure its efficacy and safety.

In this study, we characterized a unique pattern of MHV-68 infection in neuroblastoma cells. MHV-68 successfully infected and replicated in both murine and human neuroblastoma cells for a prolonged period without apparent cell deaths. Persistent infection of MHV-68 did not alter the relative growth of SHSY5Y neuroblastoma cells. Further analysis revealed that only a subset of infected cells expressed lytic proteins, although a majority of cells harbored viral genome and sustained EGFP expression. Lytic gene expression, DNA replication, and virion production were concomitantly induced after treatment with TPA and sodium butyrate, but they were blocked after treatment with GCV. Taken together, these results suggest that both productive and latent infections may be established in neuroblastoma cells, which may account for persistent infection in the brain of infected mice (Cho et al., 2009; Kang et al., 2012). Be- cause only few cell lines are available to study persistent infection of MHV-68 in vitro following de novo infection, MHV-68 infection in neuroblastoma cells may provide a valuable experimental system to investigate the various aspects of $\gamma \mathrm{HV}$ latency by using functional genomics.

\section{ACKNOWLEDGMENTS}

We thank Hye-Ri Kang for assisting data analysis. This work was supported by the National Research Foundation of Korea (NRF) grant funded by the Korean government (MEST) (NRF2012R 1A1A2004532) and the National R\&D Program for Cancer Control, Ministry of Health and Welfare, Republic of Korea (0920170).

\section{REFERENCES}

Abrams, Bae, J., Lee, D., Kim, Y.K., Gil, M., Lee, J.Y., and Lee, K.Y. (2012). Berberine protects 6-hydroxydopamine-induced human dopaminergic neuronal cell death through the induction of heme oxygenase-1. Mol. Cells 35, 151-157.

Adler, H., Messerle, M., and Koszinowski, U.H. (2001). Virus reconstituted from infectious bacterial artificial chromosome (BAC)-cloned murine gammaherpesvirus 68 acquires wild-type properties in vivo only after excision of BAC vector sequences. J. Virol. 75, 5692-5696.

Bae, J., Lee, D., Kim, Y.K., Gil, M., Lee, J.Y., and Lee, K.Y. (2012). Berberine protects 6-hydroxydopamine-induced human dopaminergic neuronal cell death through the induction of heme oxygenase-1. Mol. Cells 35, 151-157.

Bossolasco, S., Falk, K.I., Ponzoni, M., Ceserani, N., Crippa, F., Lazzarin, A., Linde, A., and Cinque, P. (2006). Ganciclovir is associated with low or undetectable Epstein-Barr virus DNA load in cerebrospinal fluid of patients with HIV-related primary central nervous system lymphoma. Clin. Infect. Dis. 42, e21-25.

Cho, H.J., Yu, F., Sun, R., Lee, D., and Song, M.J. (2008). Lytic induction of Kaposi's sarcoma-associated herpesvirus in primary effusion lymphoma cells with natural products identified by a cell-based fluorescence moderate-throughput screening. Arch. Virol. 153, 1517-1525.

Cho, H.J., Kim, S., Kwak, S.E., Kang, T.C., Kim, H.S., Kwon, H.J., Kim, Y.W., Kim, Y.S., Choi, E.K., and Song, M.J. (2009). Agedependent pathogenesis of murine gammaherpesvirus 68 infection of the central nervous system. Mol. Cells 27, 105-111.

Flano, E., Husain, S.M., Sample, J.T., Woodland, D.L., and Blackman, M.A. (2000). Latent murine gamma-herpesvirus infection is established in activated $B$ cells, dendritic cells, and macrophages. J. Immunol. 165, 1074-1081.

Flano, E., Kim, I.J., Moore, J., Woodland, D.L., and Blackman, M.A. (2003). Differential gamma-herpesvirus distribution in distinct anatomical locations and cell subsets during persistent infection in mice. J. Immunol. 170, 3828-3834.

Hwang, S., Wu, T.T., Tong, L.M., Kim, K.S., Martinez-Guzman, D., Colantonio, A.D., Uittenbogaart, C.H., and Sun, R. (2008). Persistent gammaherpesvirus replication and dynamic interaction with the host in vivo. J. Virol. 82, 12498-12509.

Kang, H.R., Cho, H.J., Kim, S., Song, I.H., Lee, T.S., Hwang, S., Sun, R., and Song, M.J. (2012). Persistent infection of a gammaherpesvirus in the central nervous system. Virology 423, 23-29.

Kovalevich, J., and Langford, D. (2013). Considerations for the use of SH-SY5Y neuroblastoma cells in neurobiology. Methods Mol. Biol. 1078, 9-21.

Lee, S., Cho, H.J., Park, J.J., Kim, Y.S., Hwang, S., Sun, R., and Song, M.J. (2007). The ORF49 protein of murine gammaherpesvirus 68 cooperates with RTA in regulating virus replication. J. Virol. 81, 9870-9877.

Lentz, T.B., Gray, S.J., and Samulski, R.J. (2012). Viral vectors for gene delivery to the central nervous system. Neurobiol. Dis. 48, 179-188

Noh, C.W., Cho, H.J., Kang, H.R., Jin, H.Y., Lee, S., Deng, H., Wu, T.T., Arumugaswami, V., Sun R., and Song, M.J. (2012). The virion-associated open reading frame 49 of murine gammaherpesvirus 68 promotes viral replication both in vitro and in vivo as a derepressor of RTA. J. Virol. 86, 1109-1118. 
Ralph, G.S., Binley, K., Wong, L.F., Azzouz, M., and Mazarakis, N.D. (2006). Gene therapy for neurodegenerative and ocular diseases using lentiviral vectors. Clin. Sci. 110, 37-46.

Ross, R.A., Spengler, B.A., and Biedler, J.L. (1983). Coordinate morphological and biochemical interconversion of human neuroblastoma cells. J. Natl. Cancer Inst. 71, 741-747.

Said, J.W., Tasaka, T., de Vos, S., and Koeffler, H.P. (1997). Kaposi's sarcoma-associated herpesvirus/human herpesvirus type 8 encephalitis in HIV-positive and -negative individuals. Aids 11, 1119-1122.

Serafini, B., Rosicarelli, B., Franciotta, D., Magliozzi, R., Reynolds, R., Cinque, P., Andreoni, L., Trivedi, P., Salvetti, M., Faggioni, A., et al. (2007). Dysregulated Epstein-Barr virus infection in the multiple sclerosis brain. J. Exp. Med. 204, 2899-2912.

Song, M.J., Hwang, S., Wong, W.H., Wu, T.T., Lee, S., Liao, H.I., and Sun, R. (2005). Identification of viral genes essential for replication of murine gamma-herpesvirus 68 using signature-tagged mutagenesis. Proc. Natl. Acad. Sci. USA 102, 3805-3810.

Stewart, J.P., Usherwood, E.J., Ross, A., Dyson, H., and Nash, T. (1998). Lung epithelial cells are a major site of murine gammaherpesvirus persistence. J. Exp. Med. 187, 1941-1951.

Suarez, A.L., and van Dyk, L.F. (2008). Endothelial cells support persistent gammaherpesvirus 68 infection. PLoS Pathogens 4 e1000152.

Terry, L.A., Stewart, J.P., Nash, A.A., and Fazakerley, J.K. (2000). Murine gammaherpesvirus-68 infection of and persistence in the central nervous system. J. Gen. Virol. 81, 2635-2643.

Weck, K.E., Kim, S.S., Virgin, H.I., and Speck, S.H. (1999). Macrophages are the major reservoir of latent murine gammaherpesvirus 68 in peritoneal cells. J. Virol. 73, 3273-3283.

Wu, T.T., Liao, H.I., Tong, L., Leang, R.S., Smith, G., and Sun, R. (2011). Construction and characterization of an infectious murine gammaherpesivrus-68 bacterial artificial chromosome. J. Biomed. Biotechnol. 2011, 926258. 